\title{
ELECTROMYOGRAPHIC ACTIVITY ASSESSMENT OF INDIVIDUALS WITH AND WITHOUT TEMPOROMANDIBULAR DISORDER SYMPTOMS
}

\author{
Juliana de Paiva TOSATO ${ }^{1}$, Paulo Henrique Ferreira CARIA ${ }^{2}$
}

1- Master's degree in Anatomy, Department of Morphology, Dental School of Piracicaba, State University of Campinas, Piracicaba, SP, Brazil. 2- DDS, MSc, PhD, Professor of Anatomy, Department of Morphology, Dental School of Piracicaba, State University of Campinas, Piracicaba, SP, Brazil.

Corresponding address: Juliana de Paiva Tosato - Rua Jacarandá, 232 - Condomínio Arujazinho IV - Arujá - SP - Phone: 551196529796. e-mail: judepaivatosato@fop.unicamp.br

Received: January 18, 2007 - Accepetd: April 17, 2007

\begin{abstract}
I

ntroduction: Temporomandibular disorders (TMD) present several signs and symptoms that hinder their correct diagnosis, which is imperative on the elaboration of a treatment plan. Over the past years, several studies have been conducted to characterize and classify TMD to better understand these disorders. Therefore, the purpose of this study was to assess the electromyographic behavior of the masseter and temporal muscles in individuals with and without myogenic, arthrogenic and mixed TMD. Method: Forty volunteers of both genders responded to the Research Diagnostic Criteria for Temporomandibular Disorders (RDC-TMD) Questionnaire, were submitted to clinical exam and underwent bilateral electromyographic exam of the masseter and temporal muscles. Results: No statistically significant difference $(p>0.05)$ was observed during the assessment of isotonic contraction. Regarding isometric contraction, pairing between the mixed TMD group and the asymptomatic subjects did not present significant difference $(\mathrm{p}>0.05)$. Comparison between the myogenic and arthrogenic TMD groups and the asymptomatic group showed statistically significant difference $(\mathrm{p}<0.05)$. The findings of the present study demonstrated alteration on the muscle contraction pattern of TMD individuals compared to that of asymptomatic patients.
\end{abstract}

Uniterms: Temporomandibular disorders; Electromyography; Temporomandibular joint; Mastication.

\section{INTRODUCTION}

Egermark-Eriksson and Ronnerman ${ }^{7}(1995)$ have stated that there are several alterations capable of affecting the temporomandibular joint (TMJ), and it is not possible to recognize one unique etiological triggering factor for temporomandibular disorders (TMD). The association of psychological, structural and postural factors originates this type of disorder, which may lead to alterations on the masticatory muscles and on the TMJ, causing varied symptomatology.

Among the characteristic multiplicity of TMD signs and symptoms, the difficulty regarding its diagnosis should be highlighted. However, its importance on the elaboration of a treatment plan should also be stressed. This topic has motivated the conduction of several studies in the past years, with the purpose of classifying TMD in order to better understand them.

The Research Diagnostic Criteria (RDC) constitute a validated diagnostic system used in TMD clinical studies, and represent an important tool on TMD investigation. The
RDC questionnaire assesses clinical, emotional and socioeconomic aspects for classifying the types of disorders ${ }^{6}$.

Another important tool used on the TMD assessment is the surface electromyography, which enables evaluating the muscle function and indicates muscle activity through the conversion of electrical signals produced by the motor unit depolarization into electrical potential ${ }^{4}$.

Although the analysis of the masticatory muscles has been performed in several studies ${ }^{2,8,9,13}$, there are few reports on myoelectric activity during mastication, particularly in cases of TMD manifestation. Thus, the electromyographic analysis under this condition is of great importance.

Due to the difficulties encountered in classifying and characterizing TMD, the goal of this study was to assess the electromyographic behavior of the masseter and temporal (anterior portion) muscles in individuals with and without myogenic, arthrogenic and mixed TMD symptoms. 


\section{MATERIALAND METHODS}

\section{Volunteers}

Forty Caucasians volunteers of both genders were enrolled in this cross-sectional study (Table 1). All volunteers received information regarding the research procedures and signed an informed consent form. The study was approved by the Ethics in Human Research Committee of the Dental School of Piracicaba, State University of Campinas, Brazil (Process number 027/2006).

\section{Inclusion and Exclusion Criteria}

To be enrolled in this study, the volunteers should meet the following criteria: be willing to participate in the study procedures, not present dental failure, have Angle's class I occlusal relationship, not be undergoing any dental or physical therapy for treatment of TMD or posture therapy and not be under any drug regimen. Volunteers aging less than 18 years old or more than 40 years old were excluded, as well as individuals with history of systemic diseases that could alter the synovial fluid, such as arthritis and arthrosis, oral breathers, subjects who have sustained facial trauma or those who met any of the above-mentioned exclusion criteria.

\section{Materials}

The following materials were used to accomplish this study: 1) Portable 8-channel Signal Conditioning Module (EMG System do Brasil, São José dos Campos, SP, Brazil) with external battery source, frequency band filter between $20-500 \mathrm{~Hz}$, amplifier with 1000x gain and standard mode rejection ratio $>120 \mathrm{~dB}$; 2) 12-bit A/D Converter (EMG System do Brasil); 3) Notebook HP connected to an EPSON CX 4500 printer; 4) Digital camera Olympus D-435; 5) Preamplified active surface electrodes with $20 \mathrm{x}$ gain, differential amplifier with bipolar input, standard mode rejection $>100 \mathrm{~dB}$ and extremity pressure button ((EMG System do Brasil); 6) Self-adhesive surface electrodes from Meditrace; 7) Ground

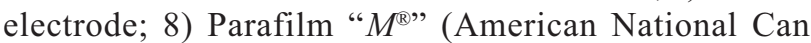
Company, Chicago, IL, USA); 9) 70\% Alcohol; 10) Cotton; 11) Rapapport Stethoscope; 12) Clamps; 13) Disposable gloves; 15) Informed Consent form; 16) Research Diagnostic Criteria for TMD (RDC-TMD) Questionnaire.

\section{Procedures}

After selection according to the inclusion and exclusion criteria, the volunteers responded to the Research Diagnostic
Criteria for TMD (RDC-TMD) Questionnaire and were submitted to the clinical exam.

Thereafter, all volunteers were submitted to the electromyographic assessment, in which four channels were utilized as follows: one for the right $\mathrm{m}$. masseter, one for the left $\mathrm{m}$. masseter, one for the anterior portion of the right $\mathrm{m}$. temporal, and one for the anterior portion of the left $\mathrm{m}$. temporal. Self-adhesive surface electrodes were utilized, along with a ground electrode to minimize artefacts. Signal acquisition started with the cleaning of the skin with cotton soaked in $70 \%$ alcohol to reduce local impedance ${ }^{5}$, followed by the placement of the electrodes guided by muscle fibers and muscle function test. Electromyographic signal was then captured in isotonic concentric and isometric contraction. For such purpose, the volunteer placed the parafilm " $M^{\mathbb{R}}$ " between the upper and lower premolars and molars. This material allows little variability on the electromyographic records ${ }^{1}$.

During the electromyographic assessment, the volunteers remained sited on a chair with back support, Frankfurt plane remained parallel to the floor, eyes opened and feet parallel against the floor. The volunteer's upper limbs were resting on the lower limbs.

After the procedure, the volunteers were assigned to 4 groups, according to the TMD classification obtained from the questionnaire and clinical exam, to further analysis of the results (Table 1): Asymptomatic Group - volunteers with no TMD and who did not present TMD signs and/or symptoms; Arthrogenic TMD Group: volunteers who presented characteristics of TMD with arthrogenic origin, such as spontaneous TMJ pain or pain during TMJ palpation, clicking, crepitus, trinities, decreased hearing acuity, mouth opening limitation or history of joint locking; Myogenic TMD Group: volunteers who presented characteristics of TMD with myogenic origin, such as headache, masticatory muscle pain during palpation or spontaneously, cervical pain; Mixed TMD Group: volunteers who presented characteristics of myogenic and arthrogenic TMD, characterizing the mixed disorder.

\section{Data Analysis}

After collecting the electromyographic records, root mean square (RMS) was used for data analysis, calculated over a fixed time-window, considering the 2nd masticatory cycle for the isotonic contraction, and the 2nd and 3rd seconds for the isometric contraction. Next, data was normalized by the peak, which presents the smaller standard

TABLE 1- Characteristics of the evaluated volunteers

\begin{tabular}{llll}
\hline & N (total) & Mean Age & Genders \\
\hline Asymptomatic & 12 & $28.16 \pm 3.63$ & $58.33 \% \mathrm{~F} / 42.66 \% \mathrm{M}$ \\
Arthrogenic & 06 & $28.5 \pm 7.81$ & $50.00 \% \mathrm{~F} / 50.00 \% \mathrm{M}$ \\
Myogenic & 09 & $27.66 \pm 6.48$ & $100.00 \% \mathrm{~F} / 0.00 \% \mathrm{M}$ \\
Mixed & 13 & $30.16 \pm 7.75$ & $92.30 \% \mathrm{~F} / 7.69 \% \mathrm{M}$ \\
\hline
\end{tabular}


deviation.

Student's t-test was used to compare the groups and identify the difference among them. Significance level of was set at $5 \%(\mathrm{p}<0.05)$.

\section{RESULTS}

The average normalized RMS values of the left masseter (LM), left temporal (LT), right masseter (RM) and right temporal (RT) muscles for the 4 groups are presented on Table 2 (isometric contraction) and Table 3 (isotonic contraction).

The average normalized RMS values were analyzed in such a way that the values of the three TMD groups were compared to those of the group with asymptomatic volunteers. No statistically significant difference was observed during the isotonic contraction assessment $(\mathrm{p}>0.05)$. Regarding the isometric contraction, pairing between the group with mixed TMD and the group with asymptomatic individuals did not present differences ( $p>0.05$ ). Comparing the myogenic TMD and the arthrogenic TMD groups to the asymptomatic group, statistically significant difference was found $(\mathrm{p}<0.05)$.

\section{DISCUSSION}

The outcome of this study demonstrated significant differences when the normalized RMS values of the myogenic and arthrogenic TMD groups were compared to those of the asymptomatic volunteers during isometric contraction. This significant difference was not observed when the mixed TMD group was compared to the asymptomatic group during isometric contraction and also when the TMD groups were compared to the asymptomatic subjects during isotonic contraction.

Analyzing the obtained results, it was possible to observe that the group of asymptomatic individuals presented greater masseter myoelectric activity in relation to the temporal muscle during isotonic and isometric contraction. The other TMD groups presented temporal muscle prevalence over the masseter muscle during isotonic and isometric contraction, with exception of the mixed TMD group, which presented greater prevalence of the masseter muscle during isotonic contraction. Scopel, et al. ${ }^{10}$ (2005), also found a prevalence of temporal muscle activation over the masseter muscles in dysfunctional individuals.

Masticatory musculature hyperactivity may contribute to the installation of TMJ internal derangement, fatigue and pain. Thus, the use of surface electromyography on the assessment of TMD may assist in the kinesiological study of muscle function during mastication ${ }^{13}$. In this study, an alteration was noted regarding the contraction pattern for the symptomatic subjects when comparing to the asymptomatic subjects during isometric contraction. This fact may be due to the presence of muscle and TMJ derangements. Bodéré, et al. ${ }^{2}$ (2005) studied the electromyographic activity of masticatory muscles in individuals with different orofacial pain conditions and asymptomatic individuals, and different activities were observed in the symptomatic and the control group, as observed in this present study.

The greater values obtained for the temporal muscle in both assessments regarding the myogenic TMD group may justify the tension-type headache, which is the distinctive

TABLE 2- Normalized RMS means ( \pm standard deviation) during isometric contraction for the 4 evaluated groups

\begin{tabular}{lcccc}
\hline & LM & LT & RM & RT \\
\hline Asymptomatic & $0.61 \pm 0.19$ & $0.55 \pm 0.23$ & $0.56 \pm 0.28$ & $0.54 \pm 0.21$ \\
Arthrogenic & $0.63 \pm 0.20$ & $0.73 \pm 0.21$ & $0.71 \pm 0.19$ & $0.75 \pm 0.23$ \\
Myogenic & $0.62 \pm 0.26$ & $0.75 \pm 0.13$ & $0.73 \pm 0.23$ & $0.77 \pm 0.18$ \\
Mixed & $0.53 \pm 0.28$ & $0.56 \pm 0.26$ & $0.47 \pm 0.28$ & $0.57 \pm 0.27$ \\
\hline
\end{tabular}

LM = Left Masseter; LT = Left Temporal; RM = Right Masseter; RT = Right Temporal.

TABLE 3- Normalized RMS means ( \pm standard deviation) during isotonic contraction four the 4 evaluated groups

\begin{tabular}{lcccc}
\hline & LM & LT & RM & RT \\
\hline Asymptomatic & $0.76 \pm 0.20$ & $0.45 \pm 0.23$ & $0.65 \pm 0.27$ & $0.50 \pm 0.22$ \\
Arthrogenic & $0.70 \pm 0.22$ & $0.70 \pm 0.25$ & $0.66 \pm 0.24$ & $0.65 \pm 0.20$ \\
Myogenic & $0.65 \pm 0.31$ & $0.78 \pm 0.19$ & $0.56 \pm 0.32$ & $0.71 \pm 0.25$ \\
Mixed & $0.45 \pm 0.31$ & $0.35 \pm 0.23$ & $0.56 \pm 0.34$ & $0.38 \pm 0.25$ \\
\hline
\end{tabular}

LM = Left Masseter; LT = Left Temporal; RM = Right Masseter; RT = Right Temporal. 
characteristic of this disorder ${ }^{3,8}$. Quinn, et al. ${ }^{11}$ (2002) explained that headaches result from ischemic areas with local nutrient deficiency, caused by sustained contractions of the temporal muscles.

Regarding the arthrogenic TMD group, the prevalence of greater temporal activation in relation to the masseter muscle may suggest compensatory mechanisms of the masticatory musculature in response to nociceptive mechanisms ${ }^{12}$.

\section{CONCLUSIONS}

According to the results obtained, it may be suggested that there was a significant difference in the electromyographic behavior of the masticatory muscles between the TMD groups and the asymptomatic group. This fact may contribute to a better understanding of the morphofunctional characteristics of the assessed muscles and facilitate diagnosis and treatment of temporomandibular disorders.

\section{ACKNOWLEDGEMENTS}

The authors would like to thank the financial support provided by CAPES to accomplish this study.

\section{REFERENCES}

1- Biasotto DA. Estudo eletromiográfico dos músculos do sistema estomatognático durante a mastigação de diferentes materiais [dissertação]. Piracicaba (SP): Faculdade de Odontologia de Piracicaba, Universidade Estadual de Campinas; 2000.

2- Bodéré C, Téa SH, Giroux-Metges MA, Woda A. Activity of masticatory muscles in subjects with different orofacial pain conditions. Pain. 2005;116(1-2):33-41.

3- Carlson CR. Psychological and physiological parameters of mastigatory muscles pain. Pain. 1998;76(3):297-307.

4- De Luca CJ. The use of surface electromyography in biomechanics. J Appl Biomech. 1997;13(2):135-63.

5- Ding R, Logemann JA, Larson CR, Rademaker AW. The effects of taste and consistency on swallow physiology in younger and older healthy individuals: a surface electromyographic study. J Speech Lang Hear Res. 2003;46(6):977-89.

6- Dworkin SF, Le Resche L. Research diagnostic criteria for temporomandibular disorders: review, criteria, examinations and specifications critique. J. Craniomandib Disord. 1992;06:302-55.

7- Egermark-Eriksson I, Ronnerman A. Temporomandibular disorder in active phase of orthodontic treatment. J Oral Rehabil. 1995;22:6138 .

8- Inoue-Minakuchi M. Intramuscular haemodynamic responses to different duration of sustained extesion in normal human masseter Arch Oral Biol. 2001;46(07):661-6.
9- Pinho JC, Caldas FM, Mora MJ, Santana-Penín U. Electromyographic activity in patients with temporomandibular disorders. J Oral Rehabil. 2000;27:985-90.

10- Scopel V, Alves GS, Urias D. An electromyographic study of masseter and anterior temporalis muscles in extra-articular myogenous TMJ pain patients compared to an asymptomatic and normal population. Cranio. 2005;23(3):194-203.

11- Quinn C, Chandler C, Moraska A. Massage therapy and frequency of chonic tension headache. Am J Public Health. 2002;92(10):165761 .

12- Sato S, Kawamura H. Natural course of non-reducing disc displacement of the temporomandibular joint: changes in electromyographic activity during chewing movement. J Oral Rehabil. 2005;32:159-65.

13- Suvinen TI, Reade PC, Kononen M, Kemprainen P. Vertical jaw separation and masseter muscle electromyographic activity: a comparative study between assymptomatic controls and patients with temporomandibular pain and dysfunction. J Oral Rehabil. $2003 ; 30: 765-72$ 